\title{
Identification of a Rare Antibody (Anti-Inb) During a Workup for a Hysterectomy
}

\author{
Sekar H, Tiwari M*, Subba B, Luckit J, Govind A, Subba R, Dhital S and Adeyemo A
}

Department of Obstetrics and Gynaecology, North Middlesex *Correspondence:

Mansi Tiwari, Department of Obstetrics and Gynaecology, North Middlesex Hospital, London, UK, E-mail: drmansitiwari@gmail. Hospital, London, UK. com.

Received: 14 November 2018; Accepted: 28 December 2018

Citation: Sekar H, Tiwari M, Subba B, et al. Identification of a Rare Antibody (Anti-Inb) During a Workup for a Hysterectomy. Food Sci Nutr Res. 2018; 1(1): 1-2.

\section{Objective}

To describe the case of a 70 year old Asian woman who presented for a hysterectomy. Pre-operative serological testing demonstrated the presence of anti-Inb red cell antibodies which warranted further investigations and planning prior to surgery.

\section{Background}

The International Society of Blood Transfusion has recently recognised 33 blood group systems representing over 300 antigens on the red cell membranes [1]. Blood grouping and cross matching is a vital pre-operative investigation prior to gynaecological surgery where blood loss may be unpredictable. To avoid a transfusionrelated reaction, thorough typing and cross-matching analysis should take place especially when rare antibodies are identified [1]. In this report, we discuss the case of a woman in whom the presence of anti-Inb red cell antibodies was identified. This group of antibodies belongs to the Indian blood group system and was first identified in 1975 [1].

\section{Case Report}

We report the case of a 70 year old woman who was referred for a hysterectomy in view of postmenopausal bleeding. She had 2 previous normal vaginal deliveries and had no history of blood transfusion in the past. In the workup for a hysterectomy, she was identified as belonging to blood group A Rhesus positive with the presence of the very rare anti-Inb antibodies. She was promptly referred to the Haematology team for further advice and management.

\section{Discussion}

Anti-Inb is found in 4\% of people of Indian origin and there have been a few case reports of haemolytic transfusion reactions
(HTR), usually precipitated by blood transfusions or immunisation through pregnancy [2]. In this patient, precautions were taken as part of a comprehensive management strategy. Firstly, the patient's pre-operative haemoglobin was optimised to over $120 \mathrm{~g} / \mathrm{L}$ with an iron infusion. Secondly, 2 units of Inb negative blood were ordered (to be on stand-by) from the National Frozen Blood Bank service in Birmingham, UK - who requires at least 24 hours' notice.

During the operation, arrangement for cell salvage was made. In the case that blood products were to be needed urgently as a result of massive haemorrhage, these could be cross-matched according to Rhesus status and anti-K antibodies, with the least incompatible unit ('most suitable') being issued. As the anti-In status of the blood products would not be known in such an event, additional close monitoring of the patient for haemolytic sequelae is paramount. The administration of immunoglobulin and steroids would be required to cover the transfusion of 'most suitable' blood units $[3,4]$.

\section{Conclusion}

As demonstrated above, this is a rare antibody. Hence, close cooperation between gynaecology, haematology and anaesthetic consultants along with the transfusion laboratory staff is imperative for a favourable outcome in the case of this complex patient. Intravenous Immunoglobulins (IVIG) and steroids have been successfully used to prevent potential transfusion reactions when giving incompatible red blood cell units and guidelines on the use of IVIG for haematological conditions have concluded that IVIG may be considered as an option for urgent situations such as a scenario of massive haemorrhage when compatible units are not available [4]. Autologous blood transfusion is also another safety measure that can be adopted [2]. 


\section{References}

1. Joshi SR. Discovery of human blood groups in India. Manipal Journal of Medical Sciences. 2018; 3: 33-36.

2. Daw Z, Padmore R, Chung A, et al. Recurrent pregnancy loss in a 33 year old woman. Laboratory Medicine. 2010; 41: 325328.

3. Robinson P, Anderson D, Brouwers M, et al. Evidence based guidelines on the use of intravenous immune globulin for haematologic and neurologic conditions. Transfusion Medicine Reviews. 2007; 21:3-8.

4. Win N, Needs M, Thornton N, et al. Transfusions of least incompatible blood with intravenous immunoglobulin plus steroids cover in two patients with rare antibody. Transfusion Practice. 2018; 58: 1626-1630. 